$10^{-x}$ is roughly $273 \times(53 / x)^{\circ}$, that is, $910^{\circ} \mathrm{A}$. at a density $10^{-16}\left(500^{\circ} \mathrm{A}\right.$. at $\left.10^{-28}\right)$.

It is evident, of course, that 'earthy' substances would condense very much more easily still.

As the error in the 'Trouton quotient' is most unlikely to be greater than 10 per cent (which makes an 8 per cent error in the index of the result), and an error of as much as 50 per cent in the specific heat makes only a 10 per cent error in that index, the estimates have presumptively considerably more than the accuracy essential to the purpose.

The subject will be discussed more fully elsewhere.

Hill Croft, Allonby, Alfred Lanck Parson.

Maryport, Cumberland. Oct. 5.

\section{Open Packing of Spheres}

IN their study of the open packing of equal spheres, Heesch and Laves' do not consider cases in which the number of spheres with which each sphere makes contact is greater than four; and at first sight it might not perhaps appear worth while to go further, for in the cubic arrangement of spheres which Barlow ${ }^{2}$ styled his "second kind of symmetry", each sphere makes contact with six, and it has a density of 0.524 .

Another and much more open arrangement with six-point contact is, however, possible; part of this structure is shown in the accompanying figure. For

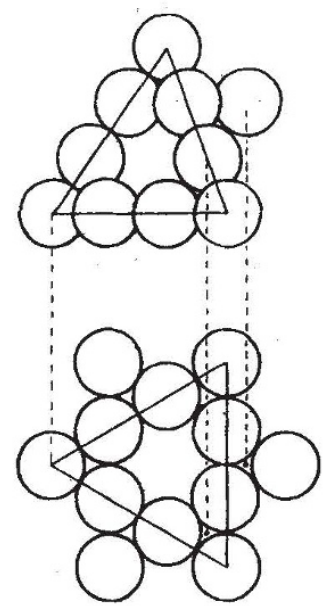
the sake of clearness only the spheres of the initial layer are indicated in the plan. The centres of these spheres lie at the vertices of a plane tessellation of equilateral triangles and hexagons. The spheres of the second layer lie over the centre of alternate triangles of the first layer. The third and all other odd-numbered sheets are congruent with the first but are translated. The fourth and all other even-numbered layers are congruent with the second but are also translated. These translations are of such a kind as cause the spheres of successive sheets to lie on the edges of regular tetrahedra, four spheres on each edge, as shown in the figure. The seventh layer lies over the first.

This structure has a density of $0 \cdot 370$, which must be reckoned remarkably low having regard to the fact that in it each sphere makes contact with as many as six others: it bears comparison with the well-known structure of diamond in which, although each sphere makes contact with only four, the density is $0 \cdot 338$.

\section{Yorkshire Museum, York. \\ Oct. 27.}

${ }^{2}$ Heesch, H., and Laves, F., Z. Krist., 85, 443 (1933).

2 Barlow, W., Nature, 29, 186 (1883).

\section{The Perithecial Stage of Didymella Lycopersici}

The perithecial stage of the fungus that causes stem rot of tomato was found and described in 1921 by Klebahn ${ }^{1}$, who named it Didymella Lycopersici. It was apparently not observed again until I found it in October 1943 on a single plant growing at Long Ashton, and since then mature perithecia have been found on three occasions*. Confirmation of the identity of the fungus has been obtained in pathogenicity experiments with single ascospore cultures.

The plant on which perithecia were found in October 1943 was one of a number that had been grown experimentally, out of doors, in pots of soil collected from a field where stem rot had severely attacked two successive crops of outdoor tomatoes. The characteristic basal stem lesion appeared early in August, but it was not until the middle of October that the plant was carefully examined. Pycnidia were then present on the upper part of the lesion; but below them, and down to soil-level, numerous perithecia were present among the disintegrating remains of the cortical tissues. At the end of December 1943, perithecia were found at or near ground-level on a number of plants at Evesham. These plants formed part of a group that had succumbed to artificial inoculation with pycnidial cultures during the summer. In the middle of July last, the perfect stage was again seen on pieces of diseased stems of affected greenhouse plants, collected at the end of May and kept moist on the surface of soil in a pot. The material had been examined from time to time but it was not until the middle of July, by which time the bulk of the cortical tissues had disappeared and only a few fructifications remained, that perithecia were found. Lastly, some diseased outdoor plants, grown from infected seed, were examined at the end of July, and one of them bore perithecia. The perithecia occurred well below ground-level, in a place that must have been approximately at soil level when the plant was in the propagating box. The cortex in this region had entirely disappeared, and scattered perithecia were attached to the woody tissues.

Klebahn ${ }^{1}$ stated that perithecia appear after overwintering. His conclusion was based on observations on pieces of diseased stem, exposed in the open dur. ing winter, on which perithecia were observed the following April. My observations show that perithecial development is not dependent on an overwintering period, but may occur at any time of year. The constant presence of perithecia near to the soil suggests that moisture supply and, possibly, soil nutrients may be factors governing their formation.

This appears to be the first record of the occurrence of perithecia of $D$. Lycopersici under natural conditions on the host. The observations have largely been confined to outdoor plants, but it is not unlikely that the perithecial stage also occurs on glasshouse plants attacked by stem rot. Notwithstanding the proved viability of the imperfect, pycnidial stage, it is possible that perithecia may play a more important part in the survival of the fungus than has hitherto been recognized.

Botanical Department,

The University, Birmingham, 15.

C. J. Hrokman.

* These observations were made while the writer was a member of the scientific staff of the Agricultural Research Council.

${ }^{1}$ Klebahn, H., Z. Pflanzenkrankheiten, 30, 1 (1921). 